\title{
Influência da Idade Materna do Período Gestacional e do Número de Gestações na Infecção pelo Papilomavirus Humano
}

\author{
Influence of Maternal Age, Pregnancy Stage and Number of Pregnancies on \\ Human Papillomavirus Infection \\ Eddie Fernando Candido Murta, \\ Maria Azniv Hazarabedian de Souza, Sheila Jorge Adad, \\ Renata Almeida Pires, Ângelo Gustavo Zucca Matthes
}

RESUMO

\begin{abstract}
Foram analisadas as informações referentes a 93 mulheres grávidas com exame Papanicolaou sugerindo infecção pelo papilomavirus humano (HPV) segundo critério de Schneider et al., com o objetivo de estudar o efeito da idade materna, do período gestacional e do número de gravidezes sobre a incidência desta infecção. O grupo controle consistiu de 93 mulheres grávidas sem sinais citológicos de infecção pelo HPV. Os resultados demonstraram que a infecção pelo HPV está associada à mulher grávida abaixo dos 20 anos de idade; entretanto, não houve relação com a idade gestacional e o número de gravidezes.
\end{abstract}

PALAVRAS-CHAVE: HPV. Infecção na gravidez. Citologia cervical. Complicações da gravidez. Colo do útero.

\section{Introdução}

A infecção pelo papilomavirus humano (HPV) é mais freqüente na gravide $z^{5} \mathrm{e}$ apresenta alta taxa de regressão após o parto ${ }^{8}$. Meisels ${ }^{4}$ demonstrou que esta infecção é mais comum na segunda metade da gestação em comparação com a primeira metade. Gopalkrishna et $\mathrm{al}^{1}$ relataram aumento da prevalência desta infecção em mulheres indianas com o aumento do número de gravidezes. Este aumento pode ser devido à imunossupressão ou à influência de fatores hormonais durante a gestação $^{3} \mathrm{Na}$ literatura existem poucos estudos sobre a relação da história obstétrica e a infecção pelo HPV, portanto, o objetivo deste trabalho é verificar os possiveis efeitos da idade, período

Disciplina de Ginecologia e Obstetrícia e de Patologia da Faculdade de Medicina do Triângulo Mineiro

Correspondência:

Eddie Fernando Candido Murta

Disciplina de Ginecologia e Obstetrícia da FMTM

Av. Getúlio Guaritá, S/N - Bairro Abadia

38025-440 Uberaba - MG

Tel: (034) 3185326, Fax: (034) 3338710

E-mail: eddiemurta@mednet.com.br gestacional e do número de gestações sobre o diagnóstico citológico da infecção pelo HPV.

\section{Material e métodos}

Analisou-se retrospectivamente as informações referentes a 93 gestantes com diagnóstico citológico de infecção pelo HPV segundo os critérios de Schneider et $\mathrm{al}^{7}$. Estes critérios são baseados na coilocitose clássica ou, pelo menos, na presença de seis dos noves critérios não clássicos, quais sejam: coilocitose leve, disqueratocitose leve, citoplasma claro, grânulos querato-hialinos, estriação citoplasmática, células paraqueratóticas, hipercrômasia celular, bi- ou multinucleação e halo claro perinuclear. As pacientes foram atendidas no período de julho de 1993 a julho de 1996. Foram colhidos dados relacionados a: idade, período gestacional (primeira ou segunda metade) e o número de gravidezes anteriores. O grupo controle consistiu de 93 mulheres grávidas atendidas no mesmo período, escolhidas aleatoriamente e que não apresentavam sinais citológicos de infecção 
pelo HPV. Na análise estatística utilizou-se o teste do $\chi^{2}$.

\section{Resultados}

Observa-se pelos dados da Tabela 1 que as alterações citológicas causadas pela infecção pelo HPV são mais freqüentes nas mulheres grávidas com idade abaixo de 20 anos de idade. Entretanto, quanto à época da gestação em que foram observadas as alterações citológicas causadas pelo HPV, se primeira ou segunda metade, não se observou diferença significante (ver Tabela 2). Na Tabela 3 observa-se que o número de gravidezes não influenciou a incidência de diagnóstico citológico do HPV.

Tabela 1 - Comparação da idade entre os grupos de mulheres grávidas com diagnóstico citológico de infecção pelo HPV (gestação + HPV) e o grupo de mulheres grávidas sem sinais citológicos de infecção pelo HPV (controle).

\begin{tabular}{lcccccc}
\hline & \multicolumn{1}{l}{ GESTAÇÃO+HPV } & \multicolumn{2}{c}{ CONTROLE } & \\
IDADE (ANOS) & $\mathrm{N}$ & $\%$ & $\mathrm{~N}$ & $\%$ & $\rho^{*}$ \\
$<20$ & 44 & 47,3 & 28 & 30,1 & $<0,05$ & $* *$ \\
$20-24$ & 26 & 28 & 21 & 22,6 & $\mathrm{NS}$ & $* * *$ \\
$>24$ & 23 & 24,7 & 44 & 47,3 & $<0,01$ & $* * * *$ \\
\hline TOTAL & 93 & 100 & 93 & 100 & & \\
\hline
\end{tabular}

$*$ Teste do $\chi^{2} ; * * \chi^{2}=5.801 ; * * * \rho=$ não significante; $* * * * \chi^{2}=10.288$

Tabela 2 - Comparação da idade gestacional entre os grupos de mulheres grávidas com diagnóstico citológico de infecção pelo HPV (gestação + HPV) e o grupo de mulheres grávidas sem sinais citológicos de infecção pelo HPV (controle).

\begin{tabular}{lcccc} 
& \multicolumn{2}{c}{ GESTAÇÃO+HPV } & \multicolumn{2}{c}{ CONTROLE } \\
SEMANAS DE GESTAÇÃO & $\mathrm{N}$ & $\%$ & $\mathrm{~N}$ & $\%$ \\
$01-20$ & 49 & 52,7 & 48 & 51,6 \\
$21-40$ & 44 & 47,3 & 45 & 48,4 \\
\hline TOTAL & 93 & 100 & 93 & 100 \\
\hline
\end{tabular}

$\rho=$ não significante (Teste do $\chi^{2}$ )

Tabela 3 - Comparação do número de gravidezes entre os grupos de mulheres grávidas com diagnóstico citológico de infecção pelo HPV (gestação + HPV) e o grupo de mulheres grávidas sem sinais citológicos de infecção pelo HPV (controle).

\begin{tabular}{lcrcc}
\hline & \multicolumn{2}{c}{ GESTAÇÃO+HPV } & \multicolumn{2}{c}{ CONTROLE } \\
NÚMERO DE GESTAÇÕES & $\mathrm{N}$ & $\%$ & $\mathrm{~N}$ & $\%$ \\
01 & 43 & 46,3 & 37 & 39,8 \\
02 & 20 & 21,5 & 21 & 22,6 \\
03 & 15 & 16,1 & 18 & 19,3 \\
04 & 15 & 16,1 & 17 & 18,3 \\
\hline TOTAL & 93 & 100 & 93 & 100
\end{tabular}

$\rho=$ não significante (Teste do $\chi^{2}$ )

\section{Discussão}

Pelos resultados acima descritos, observase uma maior incidência de alterações citológicas causadas pela infecção pelo HPV nas pacientes grávidas mais jovens. A idade é um fator importante na incidência de infecção pelo HPV nas pacientes jovens não grávidas ${ }^{2,5} \mathrm{e}$, pelos resultados deste trabalho, também o é nas mulheres grávidas.

Na gravidez há uma aumento da freqüência da infecção pelo $\mathrm{HPV}^{3,5}$. Em relação à idade gestacional, a incidência parece ser maior na segunda metade em relação a primeira metade ${ }^{1,4}$. Meisels ${ }^{4}$ analisou 29.153 citologias de mulheres grávidas, com idade gestacional conhecida, apresentando alterações citológicas compatíveis com infecção pelo HPV, demonstrando um aumento da freqüência do diagnóstico na segunda metade da gestação, sendo de 3,07\% na primeira metade e de $7,37 \%$ na segunda metade com nível de significância de $\mathrm{P}<0,001$. Gopalkrishna et al. ${ }^{1}$ estudando 100 mulheres grávidas através da hibridização do DNA do HPV, encontraram positividade para a presença do HPV em 31 (50\%) de 62 mulheres na segunda metade da gestação e em $13(34,2 \%)$ das 38 mulheres grávidas com a idade gestacional menor que 20 semanas com nível de significância de $\mathrm{P}<0,02$.

Os resultados dos autores citados acima mostraram que os sinais de infecção pelo HPV são mais freqüentes na segunda metade da gestação e confirmam estudos clínicos, citológicos e de hibridização do DNA do HPV realizados anteriormente $^{8,9}$. Estas observações podem ser explicadas pela diminuição de função do sistema de defesa imunológica devido aos altos níveis de estrógeno e progesterona que podem interferir no sistema regulatório da replicação viral ${ }^{6}$. Entretanto, no nosso trabalho não houve diferença estatística na freqüência de alterações citomorfológicas de infecção pelo HPV na citologia entre a primeira e a segunda metade da gestação. Este achado difere dos relatados na literatura e pode ser explicado pelo fato que o vírus está presente nestas pacientes e com o evoluir da gravidez há uma maior expressão clínica e laboratorial da infecção.

Em relação ao número de gravidezes, nossos resultados mostraram uma diminuição da freqüência da infecção pelo HPV com o maior número de gravidezes. Gopalkrishna et $\mathrm{al}^{1}$ demonstraram uma maior freqüência da infecção pelo vírus com o aumento do número de gravidezes na Índia e justificaram este achado pelo casamento em idade mais jovem das mulheres naquele país e pelo alto número de gravidezes, que na sua maioria são prenhezes subseqüentes, sem intervalo de 
tempo, facilitando a replicação do vírus com mais freqüência e perpetuando a infecção. Concluímos que os sinais citológicos da infecção pelo HPV nas pacientes grávidas por nós estudadas está relacionado com idade abaixo dos 20 anos de idade, entretanto não encontramos relação com a idade gestacional e o número de gravidezes.

\section{SUMMARY}

We have analyzed 93 pregnant women with Papanicolaou smears suggesting human papillomavirus (HPV) infection (Schneider et al's criteria) with the purpose of studying the effect of maternal age, stage of pregnancy and number of pregnancies on the incidence of this infection. The control group consisted of 93pregnant women with Papanicolaou smears not suggestive of HPV infection. The results demonstrated that HPV infection is associated with pregnant women under 20 years of age, but no differences were found regarding stage and number of pregnancies.

KEY WORDS: HPV. I nfection in pregnant women. Cervical cytology

\section{Referências bibliográficas}

1. Gopalkrishna V, Murthy NS, Sharma JK, Roy M, Das DK, Luthra UK \& Das BC. Increased human papillomavirus infection with the increasing number of pregnancies in Indian women. J Infect Dis 1995; 171 : 254-5.
2. Hildesheim A, Gravlu P, Schillman MH. Determinants of genital human papillomavirus infetion in lowincome women in Washington D.C.. Trans Dis 1993; 20: 279-285.

3. Kiviat NB, Koustsky LA, Critchlow CW. Comparison of Southern transfer hybridization for detection of cervical human papillomavirus infection with types, 6, 11, 16, 18,31,33 and 35. Am J Clin Pathol 1990; 94: 561-5.

4.Meisels A. Cytologic diagnosis of human papillomavirus: influence of age and pregnancy stage.

Acta Cytol 1992; 3636 480-2.

5. Murta EFC, Souza MAH, Lombardi W, Borges LS. Aspectos epidemiológicos da infecção pelo papilomavirus humano. J Bras Ginecol 1997; 107: 95-9.

6. Ponta H, Kenedy N, Scroch P, Hynes NE, Groner B. The hormonal response region in the mouse mammary tumors virus long termonal repeat can be dissociated from the proviral promoter and has enhancer propertiers. Proc Natl Acad Sci USA 1985; 82: 1020-4.

7. Schneider A, Meinhardatr G, de Villers EM \& Gismmann L. Sensitivity of the cytologic diagnosis of cervical condyloma in comparison with HPVDNA hybridization studies. Diagn Cytopathol 1987; 3: 250-5.

8. Schneider A, Hotz M, Gissmann L. Increased prevalence of human papillomaviruses in the lower genital tract of pregnant women. Int $\mathbf{J}$ Cancer 1987: 156: 904-11.

9. Woodruff JD, Peterson WF. Condylomata acumi-nata of the cervix. Am J Obstet Gynecol 1958; 75: 1354-62 